\title{
The ubiquitous mechanism accelerating cosmic rays at all the energies
}

\author{
Antonio Codino ${ }^{a, *}$ \\ ${ }^{a}$ University of Perugia and INFN, \\ Via A. Pascoli, Perugia, Italy \\ E-mail: antonio.codino@pg.infn.it
}

The mechanism accelerating cosmic rays in the $\mathrm{M}$ ilky $\mathrm{W}$ ay Galaxy and galaxy clusters is identified and described. The acceleration of cosmic rays is a purely electrostatic process which operates up to the maximum energies of $10^{23} \mathrm{eV}$ in galaxy clusters. Galactic cosmic rays are accelerated in a pervasive electrostatic field active in the whole Galaxy except in restricted regions shielded by interstellar and stellar plasmas as, for instance, the region occupied by the solar system.

It is proved that the energy spectrum of the cosmic radiation in the Milky Way Galaxy, in the region where the solar system resides, has a constant spectral index comprised between 2.64-2.68 and the maximum energies of Galactic protons are $3.0 \times 10^{19} \mathrm{eV}$. The agreement of these results with the experimental data is discussed in detail and underlined.

The various physical processes that maintain the stability of the electrostatic structure in the Milky Way Galaxy are the same that generate the Galactic magnetic field. Accordingly, the intensity, orientation and direction of the Galactic magnetic field are evaluated. The results of the calculation are compared with the observational data, optical and mostly radio astronomy data. The accord of the intensity, orientation and direction of the observed magnetic field with calculation is excellent.

$37^{\text {th }}$ International Cosmic Ray Conference (ICRC 2021)

July 12 th - 23rd, 2021

Online - Berlin, Germany

\footnotetext{
${ }^{*}$ Presenter
} 


\section{Introduction}

Experimental and theoretical studies have attempted for many decades to identify the mechanisms and cosmic sites where the atomic nuclei of the quiescent galactic matter are converted into cosmic rays, namely, ions of ultrarelativistic energies. Particle acceleration mechanisms have been conceived in 1954 by Enrico Fermi [1, 2] and have survived, with a number of variants, until the present days.

Such mechanisms are not adequate to account for the experimental data and particularly, the differential energy spectrum of the cosmic radiation, $J$, which extends up to the energy of $3 \times 10^{20}$ $e V$ [3-6]. This spectrum as it is well known, in restricted energy bands, can be expressed by a piecewise power law, $J=a / E^{\gamma}$ where $E$ is the cosmic ray energy, $\gamma$ is the spectral index and $a$ is a constant. For example, in the interval $10^{10}-2 \times 10^{15} \mathrm{eV}$ from a variety of experimental data it turns out, $a=9574$ particles $/ m^{2} s s r \mathrm{GeV}^{1.67}$, an index $\gamma$ of 2.67 [7] and a particle rate of one particle $/ m^{2} s \mathrm{sr} \mathrm{GeV}$ at the energy of $30.9 \mathrm{GeV}$. At this arbitrary energy the intensity is not reduced by the solar modulation.

A new and universal mechanism is proposed in this paper which explains the acceleration of cosmic rays from the energy of the quiescent matter up to $10^{23} \mathrm{eV}$ and beyond. Energies of the quiescent matter are those of the ionization of atomic nuclei, crudely in the range $5 \mathrm{eV}-10 \mathrm{keV}$ along with those of cold interstellar matter down to energies of $10^{-3} \mathrm{eV}$.

\section{The electrostatic field residing in the Milky Way Galaxy}

he observed energies of extrasolar cosmic rays extend from about $30 \mathrm{MeV}$ to $2 \times 10^{20} \mathrm{eV} \mathrm{[3-6].}$ The observed spectral index $\gamma$ is measured to be in the range 2.6-2.7 for all cosmic nuclei with energies below $10^{15} \mathrm{eV}$. Once the energy spectrum is known, the total kinetic energy transported by the cosmic rays can be determined. The kinetic energy per unit volume turns out to be about 1.1 $\mathrm{eV} / \mathrm{cm}^{3}$. Notice that in the evaluation of the energy density of cosmic rays the maximum energy of the spectrum is not critical since the major fraction of the energy is concentrated between 0.5 and $10 \mathrm{GeV}$. Accordingly, the energy of cosmic rays per unit volume is an observed, empirical quantity, at least a secure order of magnitude.

Cosmic rays are charged particles and, therefore, the simplest, conceivable means they acquire the enormous amount of kinetic energy they store might originate from an electric field. In this case the problem to resolve is to recognize where this electric field is located, how it is sometimes hidden in the cosmic ambient and how the acceleration of nuclei, electrons, positron and antiprotons would take place.

\section{The total negative charge stored in spiral galaxies}

If the kinetic energy of cosmic rays would originate from an electrostatic source, the order of magnitude of the amount of electric charge which would generate the electrostatic potential $V_{e}$ and the related electrostatic energy $U_{e}$ can be determined by the Virial Theorem. As cosmic rays are a population of charged particles, when they are immersed in an electrostatic field they respond via the Coulomb law which depends on the square of the particle distance between particle pairs. 
This theorem dictates that after sufficiently long time, the average kinetic energy $\bar{T}_{c r}$ of a system of particles endowed with electric charge equals the half of the electrostatic potential energy, $\bar{U}_{e}$, namely :

$$
\bar{U}_{e}=-2 \bar{T}_{c r}
$$

For sake of simplicity let us imagine a sphere of radius $r_{g}=15 \mathrm{kpc}$ and a volume $V=4 / 3 \pi$ $r_{g}^{3}=415 \times 10^{66} \mathrm{~cm}^{3}$ representative of the volume of a typical galaxy like the Milky Way Galaxy albeit this celestial body is not spherical. Let $Q_{c r}$ be the electric charge of cosmic rays which is globally positive, and it is carried by cosmic nuclei. The electric charge conservation in a finite volume requires an equal amount of negative charge in the Galactic volume denoted here by $Q_{w}$, and consequently, the following relation holds: $\quad Q_{c r}+Q_{w}=0 \quad$ regardless of the way cosmic rays are accelerated. The negative charge $Q_{w}$ ( $w$ is for widow electron) is stored by electrons of the quiescent Galactic matter being these particles the only stable and available.

Preliminarily, let us admit that the negative electric charge $Q_{w}$ is uniformly distributed in the spherical volume of radius $r_{g}$ while the positive charge $Q_{c r}$ has a spherical symmetry with a decreasing radial density of the form $k / r$ appropriate for cosmic rays emanating from a finite source where $k$ is an appropriate constant. The dependence $1 / r$ is a characteristic feature of the diffusive motion and it extends beyond $r_{g}$ up to a maximum distance $R$ (for instance $R=20 r_{g}$ ). Fig. 1 highlights what has been imagined up here showing, qualitatively, the positions of the negative and positive electric charges of cosmic rays while neutral matter is omitted for simplicity.

In the specified conditions, the total electric charge $q_{g}$ within the sphere of radius $r_{g}$ is : $q_{g}=\left[Q_{w}-Q_{c r}\left(r_{g}\right)\right]=Q_{w}\left(r_{g} / R\right)^{2}$. The electrostatic energy $E_{e}$ contained in the same sphere amounts to: $E_{e}=Q_{w}^{2} /\left(4 \pi \epsilon_{0} R\right)\left(-l / 4+l^{3} / 6+l^{5} / 10\right) \quad$ being $l \equiv\left(r_{g} / R\right)$. Since $l$ is a small number, the terms $\left(l^{3} / 6+l^{5} / 10\right)$ can be neglected and it results : $\quad E_{e}=Q_{w}^{2} r_{g} /\left(16 \pi \epsilon_{0} R^{2}\right)$. A first good approximation is to apply equation (1) to the volume $V$ and the result is: $\bar{U}_{e} V=2 \bar{T}_{c r} V=$ $E_{e} \quad$ and hence the charge $q_{g}$ is:

$$
q_{g}=\pi r_{g} R\left[(128 / 3) \epsilon_{0} \bar{T}_{c r}\right]^{1 / 2}
$$

Substituting the values of $r_{g}, R$ and $\bar{T}_{c r}$ in equation (2) the resulting charge is $q_{g}=1.0910^{32}$ Coulomb (hereafter $C)^{1}$.

\section{On the intensity of the radial magnetic field of the Milky Way Galaxy}

Gas, dust and stars of the Milky Way Galaxy regularly rotate about the Galactic center with typical velocities in the range $230-250 \mathrm{Km} / \mathrm{s}$. These velocities, as it is well known, are measured with great precision. Measurements of the circular velocities of $230-250 \mathrm{Km} / \mathrm{s}$ of clouds and stars span the distances 300-400 $p c$ from the Galactic center up to $15000 p c$ and beyond.

\footnotetext{
${ }^{1}$ The analytical calculation of the electric field in a sphere of volume $V$ is straightforward and partially justifies the adoption of this geometrical form. A disk-shaped volume with half thickness $h=125 \mathrm{pc}$ and radius $r_{g}=15 \mathrm{kpc}, a$ more appropriate surrogate of the disk volume of the Milky Way Galaxy yields an electric charge $q_{g}$ comparable to that obtained with the equation (2). Detailed calculation of the electrostatic field and related potential in a flat disk, surrounded by a positively charged larger halo, are given in Chap. 9 of ref. [8].
} 


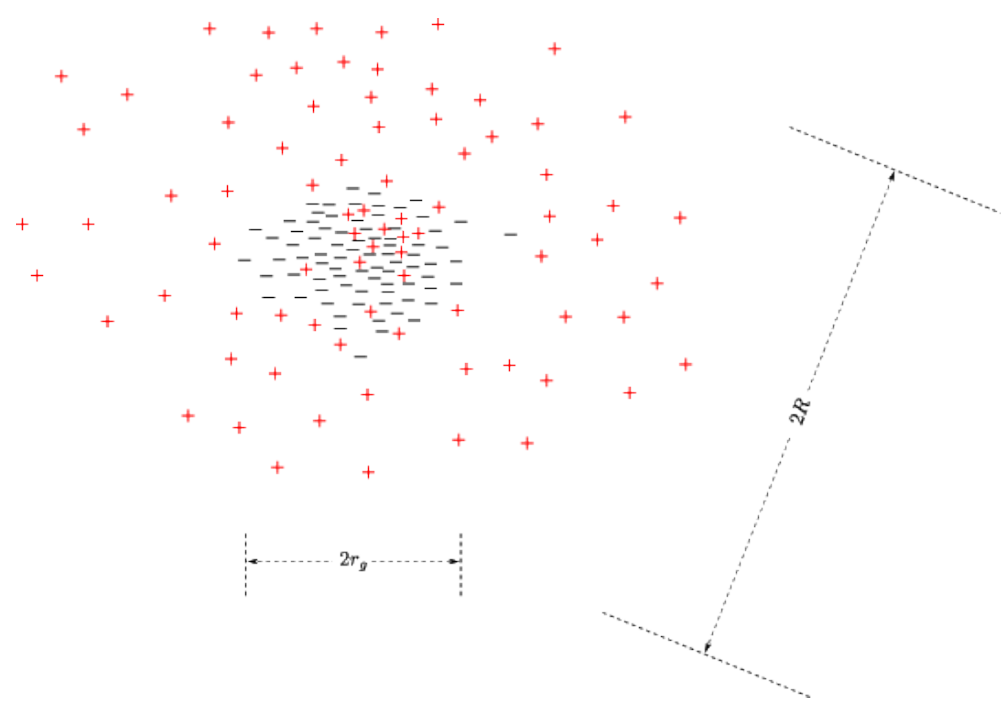

Figure 1: Qualitative illustration of the positions of the electric charges transported by cosmic rays in a spherical globe of maximum size $2 R$. The negative charges of the electrons of the quiescent galactic matter are represented by negative signs and they are confined in a region of size $2 r_{g}$ while the positive charges of protons, nuclei and positrons represented by plus signs extend up to $2 R$. High energy electrons, unlike protons and nuclei, radiate synchrotron (curvature) light and cannot diffuse over large distances from the globe of characteristic size $2 r_{g}$. Cosmic nuclei propagate via a diffusive-alternate motion (see Appendix B.3 and fig. 81 of ref. [8]) forming a halo of positive charge whose density decreases with the radial distance $r$ in the range $0 \leq r \leq R$. The different distributions of the negative (quiescent electrons) and positive (cosmic nuclei) charges generate an electrostatic field denoted $\vec{E}_{g}$. The cartoon is not at scale and ignores many details discussed elsewhere [8].

An electric charge in a circular motion produces a characteristic magnetic field. Let us assume that spiral galaxies retain a negative electric charge of the same order of magnitude of $q_{g}$ and simplify the form of the Milky Way Galaxy by a rotating rigid disk. Subdivide such a disk in $n$ circular coronas, as shown in fig. 2-b. Any corona is endowed with an equal amount of electric charge $q_{n}=q_{g} / n$. In these conditions the order of magnitude of the magnetic field originated by the circular motion can be straightforwardly estimated.

The magnetic field strength at the center of the circular coil of radius $r_{n}$ traversed by the current $i_{n}$ is, $\quad \mu_{0} i_{n} / 2 r_{n}$ the direction of the magnetic field is normal to the disk plane. The total field strength $\vec{B}$ results from the sum of all currents $i_{n}$ in the $n$ coils, $\vec{B}=\sum_{n} \vec{B}_{n}=\left(\sum_{n}\right.$ $\left.\mu_{0} i_{n} / 2 r_{n}\right) \vec{u}_{z} \quad$ being $r_{n}$ the radius of the $n$-th circular coil, $\vec{u}_{z}$ the unit vector of the $z$ axis and $i_{n}=q_{n} / T_{n}$ with $T_{n}$ the revolution period of the electric charge $q_{n}$ around the Galactic center. For instance, with $n=10$, charges $q_{n}$ proportional to the coil volumes, equal velocities of $245 \mathrm{Km} / \mathrm{s}$ for all the coronas which are placed at the radial distances $\left(r_{n}-r_{n-1}\right) / 2$, it turns out a magnetic field strength of $1.04 \mu G$.

The order of magnitude of such a field strength equals that of the regular magnetic field measured in the Milky Way Galaxy, in Andromeda and in many other spiral galaxies. A precise evaluation of the intensity, orientation, and direction of the Galactic magnetic field is reported in the magnetic chapters of ref. [8]. 


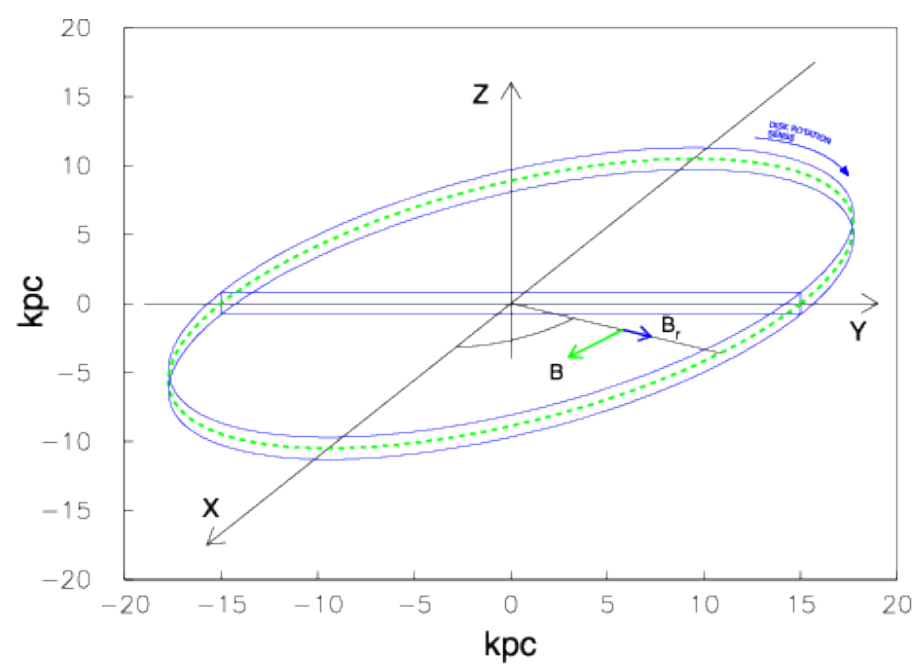

2-a

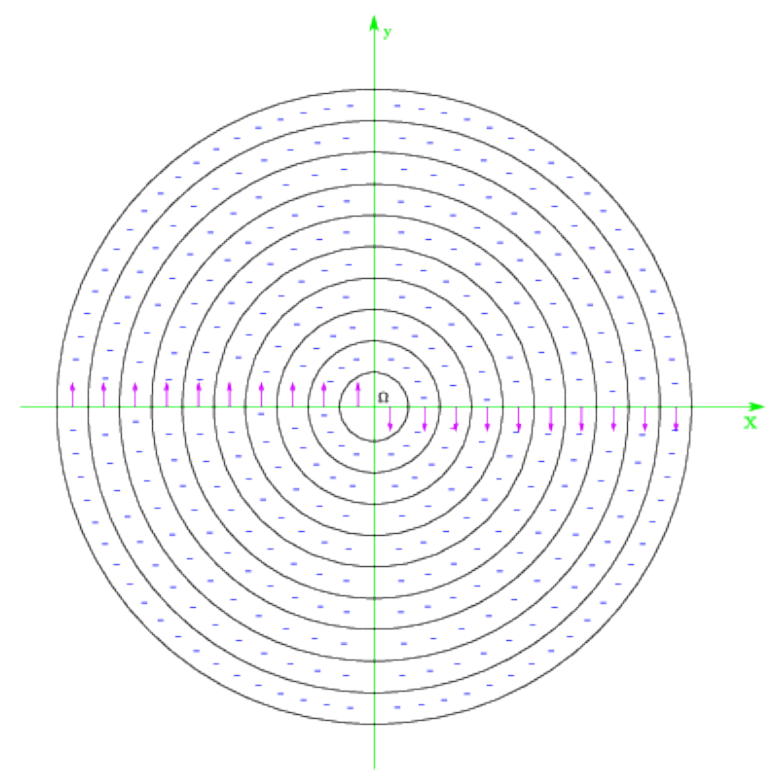

$2-\mathrm{b}$

Figure 2: Illustration of a magnetic field generated by a rigid disk rotating about the $z$ axis at the velocity of $245 \mathrm{Km} / \mathrm{s}$. The height $h$ of the disk is $200 \mathrm{pc}$ and the radius $r_{g}$ is $15000 \mathrm{pc}$. The negative charge $q_{g}$ of $1.09 \times 10^{32} C$ is uniformly distributed in the disk volume. 2- $a$ The reference system in the disk adopted in this work, the orientation of the axes $x y$ and $z, \quad$ the sense of rotation seen from the north Galactic pole (blue arrow), and the Galactic midplane (annular, green, dashed curve) are shown. It is also indicated the orientation of the $\phi$ angle which defines the azimuthal component $\vec{B}_{\phi}$ of the Galactic magnetic field. 2-b The 10 concentric coronas resulting from the partition of the disk volume used in the calculation of the radial magnetic field. The violet arrows indicate the velocity orientation of the 10 coronas seen from above and the minus signs denote the negative charge of the rotating quiescent electrons. 
In summary, taking advantage of the Virial Theorem expressed by equation (1), the density of kinetic energy of cosmic rays per unit volume of $1.1 \mathrm{eV} / \mathrm{cm}^{3}$ enables to determine the amount of electric charge that generates the corresponding electrostatic potential $V_{e}(r)$. The reasoning up to this point, rests on a simple and natural assumption. The main mode any electric charges like cosmic rays undergo acceleration is by an electric field.

It has been realized that the magnetic field strengths in spiral galaxies fall in the correct order of magnitude under the assumption that the electric charge $Q_{w}$ rotates around the Galactic center, as calculated above. Thus, the assumption of an electric charge $Q_{w}$ in the Galaxy already introduced with independent arguments (Seg. 2.1), deserves an adequate exploration and justifies the quest for the mechanism accelerating cosmic rays along the electrostatic avenue.

\section{Maximum energies of Galactic cosmic rays}

Proceeding further, the same amount of charge $q\left(r_{g}\right)$ has its distinctive electrostatic potential $V_{e}(r)$ which at the surface of sphere $r=r_{g}$ is $V_{e}\left(r_{g}\right)=q_{g} / 4 \pi \epsilon_{0} r_{g} \quad$ and with $r_{g}=15 \mathrm{kpc}$ and $q_{g}$ $=1.097 \times 10^{32} C$ gives $21.3 \times 10^{20} \mathrm{~V}$. Let us denote $V_{e}^{m a}$ the maximum value of the potential on the spherical surface at $r_{g}$. A cosmic proton of charge $q$ moving from the peripheral zone, $r=r_{g}$ to the center of the sphere $r=0 p c$, would gain the energy $q V_{e}\left(r_{g}\right)$ which is $21.3 \times 10^{20} \mathrm{eV}$.

The Milky Way Galaxy is not spherical but disk shaped and, a more realistic halo of positive electric charge, for example $R=r_{g}=15 \mathrm{kpc}$ along with a charge $q_{g}=2.5 \times 10^{31} \mathrm{C}$ (evaluated with more precise geometrical Galactic parameters in Chap. 9 of ref. [8]), slightly less than that reckoned in Seg. 2.1, would give an electrostatic potential on the disk rim at $r_{g}=15 \mathrm{kpc}$ of $-3.64 \times 10^{20} \mathrm{~V}$. At the radial distance of the Sun at $r_{s}=8.5 \mathrm{kpc}$ the potential is, $-5.40 \times 10^{20} \mathrm{~V}$ (Seg. 8.1 of ref. [8]). In these conditions the maximum kinetic energies of protons are $1.7 \times 10^{20}$ $\mathrm{eV}$.

It results that protons wander in the Milky Galaxy with maximum energies in the range (3-8) $\times 10^{19} \mathrm{eV}$. This energy interval is close to the maximum energies of cosmic rays measured at Earth of about $3 \times 10^{20} \mathrm{eV}$ [3-6]. These energies coincide with the energy band (2-6) $\times 10^{19} \mathrm{eV}$ where a rapid flux drop (cutoff) relative to the extrapolated power-law smooth spectrum has been observed [9]. The cutoff energy region has been interpreted as unmistakable, distinctive signature of maximum energies of Galactic protons (see Chap. 13 of ref. [8]). Thus, again experimental data [3-6] consistently revisited for the correct energy scale [10,11] are compatible with the crude but essential evaluations just mentioned.

Besides the concordance in the order of magnitude of the galactic magnetic field with that derived in Seg. 2.2 based on the circular motion of the charge $q_{g}$ stored in the disc, an additional, independent agreement of order of magnitude, emerges from the comparison of the maximum energies of the cosmic rays and the maximum electrostatic potential energy per particle $Z q V_{e}^{m a}$. 
The sentiment is that the above concordance in two disparate areas is not a mere, casual coincidence since these two physical quantities, maximum energy of cosmic protons and the intensity of the Galactic magnetic field have very distinctive, unmistakable values, e.g. $2.7 \times 10^{19}$ $\mathrm{eV}$ and $10^{-10} \mathrm{~T}$. These two incredibly disparate numerical figures communicate by the electrostatic field resident in the Galaxy if the intuition conceived at the beginning of this work is correct. Let us remind that the differential intensity of the cosmic radiation $J(E)$ span 31 orders of magnitude over 10 energy decades (see fig. 46 in ref. [8]).

\section{Electrostatic field intensity in the Milky Way Galaxy}

As it is well known the electrostatic energy of a system of electric charges can be regarded not only as the total work necessary to displace the electric charges from very large distances to the positions they actually occupy in the system but, also, as energy density of the electrostatic field which permeates the space around the charges. Accordingly, the average intensity of the electrostatic field $\bar{E}_{g}$ in the spherical volume $V$ can be crudely estimated by the equation :

$$
E_{e}=\frac{1}{2 V} \int \epsilon_{0} E_{g}^{2} d V=\frac{\epsilon_{0}}{2 V}\left(\bar{E}_{g}\right)^{2} V=\frac{\epsilon_{0}}{2}\left(\bar{E}_{g}\right)^{2}
$$

being $d V$ the infinitesimal volume element, $\vec{E}_{g}$ the electrostatic field at any point in space and $\bar{E}_{g}$ its average value. The electrostatic energy $E_{e}$ also follows from equation (1), $E_{e}=2$ $\bar{T}_{c r} V$ which substituted in the first member of equation (4) gives an estimate of the average value of the electrostatic field. The result is :

$$
\bar{E}_{g}=\left(4 \bar{T}_{c r} / \epsilon_{0}\right)^{1 / 2}
$$

giving $\bar{E}_{g}=0.28 \mathrm{~V} / \mathrm{m}$. 
Detailed calculation of the shape and intensity of the Galactic magnetic field $\vec{B}_{g}$ requires the knowledge of the dynamics of cosmic rays and quiescent charged particles in the Milky Way Galaxy and overwhelms the size of this conference paper. Similarly, the spectral index $\gamma$ of 2.65 of the cosmic-ray spectrum is calculated elsewhere [8]. Both the index $\gamma$ and $\vec{B}_{g}$ are found in agreement with the experimental data as evidenced in [8].

\section{References}

[1] E. Fermi, On the Origin of the Cosmic Radiation, Physical Review 75, 1169-1174.

[2] E. Fermi, Galactic Magnetic field and the origin of the Cosmic radiation, The Astrophysical Journal (hereafter ApJ), 1949, 119, 1-6.

[3] N. Hayashida et al. (Coll. AGASA), Observation of a very Energetic cosmic ray well beyond the predicted 2.7 K cutoff, Physical Review Letters, 73, 3491-3494.

[4] D. J. Bird et al. (Coll. Fly's Eye), 1994, The cosmic-ray energy spectrum observed by Fly 's Eye, ApJ, 424, 491-502.

[5] P. Tinyakov et al. (Coll. Telescope Array), 2014, Latest results of the Telescope Array, Nuclear Instruments and Methods in Physics Research, A 742, 29-34.

[6] V. Verzi et al., 2017, Measurement of energy spectrum of ultra-high Energy cosmic rays, [arXiv: astro-ph/1705.09111v1], 25 May 2017; also in Progress in Theoretical and Experimental Physics (2017).

[7] J. P. Boyle et al. (Coll. TRACER), 2011, New measurements of the composition and energy spectra of cosmic-ray nuclei with TRACER, Proc. 31st International Cosmic Ray Conference (hereafter ICRC), work 707, Beijing, China.

[8] A. Codino, 2020, The Ubiquitous mechanism accelerating cosmic rays at all the energies, Società Editrice Esculapio, Bologna, Italy.

[9] R. U. Abbasi et al., 2004, Measurements of the Flux of Ultrahigh energy Cosmic Rays from Monocular Observations by the High Resolution Fly' s Eye experiment, Physical Review Letters, 92, 151101 (4 pp).

[10] A. Codino, 2013, The Absence of the GZK Depression in the Energy Spectrum of the Cosmic Radiation, work 748, 33nd ICRC, Rio de Janeiro, Brasil.

[11] A. Codino, 2019, About the consistency of the energy scales of past and present instruments detecting cosmic rays above the ankle energy, [arXiv.1710.06659v1] (astro/ph.HE), 8 July 2017, submitted to Astronomy and Astrophysics. 